\title{
PRIME VON NEUMANN REGULAR RINGS AND PRIMITIVE GROUP ALGEBRAS
}

\author{
JOE W. FISHER AND ROBERT L. SNIDER
}

\begin{abstract}
Kaplansky posed the following question: Are prime von Neumann regular rings primitive? We show that with a certain countability condition the answer is affirmative. This is used to simplify and clarify earlier work on the existence of primitive group algebras.
\end{abstract}

In [7] Kaplansky posed the following question: Are prime von Neumann regular rings primitive? Our main theorem in $\S 1$ asserts that a prime von Neumann regular ring with a countable cofinal subset of ideals is indeed primitive. From this it follows that Kaplansky's question has an affirmative answer for each of the following classes of rings: (1) countable rings, and (2) rings with the descending chain condition on two-sided ideals.

We call locally Artinian a ring which is the union of an ascending sequence of left Artinian subrings. In $\$ 2$ we establish that a semisimple locally Artinian ring is primitive if and only if it is prime. This elucidates and extends earlier work of Formanek and Snider [5] and Passman [11] on the existence of primitive group algebras. Moreover, we apply it to produce new primitive group algebras by showing that the group algebra over any field of the group of permutations on an infinite set which move only finitely many elements is primitive.

1. Prime von Neumann regular rings. Let $R$ be a prime ring. The unadorned word "ideal" will always mean "two-sided ideal". Under the partial order of inclusion the set $S$ of nonzero ideals of $R$ is a directed set since $I, J$ in $S$ implies $I \cap J$ in $S, I \cap J \subseteq I$, and $I \cap J \subseteq J$. We say that $R$ has a countable cofinal subset of ideals if there exists a countable subset $C$ of $S$ so that for each $A$ in $S$ there is an $I$ in $C$ such that $I \subseteq A$. We will be considering a ring $R$ in which each nonzero right ideal contains a nonzero idempotent. Note that this is equivalent to each nonzero left

Presented to the Society, April 28, 1973 under the title Prime regular rings; received by the editors February 16, 1973.

AMS (MOS) subject classifications (1970). Primary 16A26, 16A30; Secondary 16A12, 16A20, 16A32.

Key words and phrases. Prime von Neumann regular rings, primitive group rings, locally Artinian rings, generalized polynomial identity, locally finite groups.

(c) American Mathematical Society 1974 
ideal of $R$ contains a nonzero idempotent. We denote the Jacobson radical of $R$ by $J(R)$ and call $R$ semisimple if $J(R)=0$.

THEOREM 1.1. If $R$ is a prime ring in which each nonzero right ideal contains a nonzero idempotent and if $R$ has a countable cofinal subset of ideals, then $R$ is both left and right primitive.

Proof. We will prove that $R$ is left primitive by producing a maximal left ideal of $R$ which contains no nonzero ideals of $R$. From the left-right symmetry of the hypotheses it will follow that $R$ is also right primitive.

Let $\left\{I_{n}: n=1,2,3, \cdots\right\}$ be a countable cofinal subset of ideals of $R$. Let $e_{1}$ be a nonzero idempotent in $I_{1}$. Since $R$ is a prime ring, $e_{1} R \cap I_{2}$ is a nonzero right ideal and thus contains a nonzero idempotent $e_{2}$. Likewise, $e_{2} R \cap I_{3}$ contains a nonzero idempotent $e_{3}$. By continuing in this fashion we obtain a sequence of idempotents $\left\{e_{n}: n=1,2,3, \cdots\right\}$. Note that $R\left(1-e_{1}\right) \subseteq R\left(1-e_{2}\right) \subseteq R\left(1-e_{3}\right) \subseteq \cdots$ and hence $L=\sum_{n=1}^{\infty} R\left(1-e_{n}\right)$ is a proper left ideal of $R$; otherwise,

$$
e_{1}=r_{1}\left(1-e_{1}\right)+r_{2}\left(1-e_{2}\right)+\cdots+r_{k}\left(1-e_{k}\right)
$$

from which it follows that $e_{n}=e_{1} e_{n}=0$ for $n \geqq k$. Moreover, $L$ is a modular left ideal since $r-r e_{1}$ in $L$ for each $r$ in $R$. Consequently, $L$ can be extended to a modular maximal left ideal $K$ which contains $L$. Finally we claim that $K$ contains no nonzero ideals of $R$. If $K$ contains a nonzero ideal $I$, then $K=K+I \supseteq L+I$. Since $\left\{I_{n}: n=1,2,3, \cdots\right\}$ is cofinal, there exists an $n$ such that $I \supseteq I_{n} \supseteq\left\{e_{n}\right\}$. Therefore $K \supseteq L+I \supseteq R\left(1-e_{n}\right)+R e_{n}=R$, which is a contradiction.

REMARK. In the above proof we have assumed that $R$ has a unity element to simplify notation; however, the standard modifications can be made to yield a proof without unity.

COROLlARY 1.2. If $R$ is a prime von Neumann regular ring which has a countable cofinal subset of ideals, then $R$ is primitive.

Proof. Evident.

COROLlaRY 1.3. If $R$ is a countable prime von Neumann regular ring, then $R$ is primitive.

Proof. The principal ideals form a countable cofinal subset.

It is well known that a prime von Neumann regular ring with a polynomial identity is primitive. We conclude this section by proving more generally that a prime regular ring with a generalized polynomial identity is primitive. In order to do this we relate the primitivity of $R$ to the primitivity of the Martindale central closure $S$ of $R$. For definitions and terminology see Martindale [9]. 
THEOREM 1.4. If $R$ is a prime von Neumann regular ring for which the central closure $S=R C$ of $R$ is primitive, then $R$ is primitive.

Proof. Let $N$ be a faithful irreducible right $S$-module. We claim that $N$ is also a faithful irreducible right $R$-module and hence $R$ is primitive. Let $0 \neq v \in N$ and $w \in N$. Then there exists $\sum x_{i} c_{i}$ in $S$ with $x_{i} \in R, c_{i} \in C$ such that $v\left(\sum x_{i} c_{i}\right)=w$. It can be shown that there is a nonzero ideal $I$ of $R$ with $c_{i} I \subseteq R$ for each $i$. Now $N$ is also a faithful irreducible $I C$ module. Hence there exists $\sum y_{j} d_{j}$ with $y_{j} \in I, d_{j} \in C$ such that $v\left(\sum y_{j} d_{j}\right)=v$. Since $R$ is regular, $\sum R y_{j}=R y$ for some $y \in R$ with $y^{2}=y$. Whence

$$
\begin{aligned}
w & =v\left(\sum y_{j} d_{j}\right)\left(\sum x_{i} c_{i}\right)=v\left(\sum y_{j} y d_{j}\right)\left(\sum x_{i} c_{i}\right) \\
& =v\left(\sum y_{j} d_{j}\right)\left(\sum y x_{i} c_{i}\right)=v\left(\sum\left(c_{i} y\right) x_{i}\right) .
\end{aligned}
$$

However, $c_{i} y \in R$ for each $i$ yields that $\sum\left(c_{i} y\right) x_{i}$ in $R$. Thus $N$ is an irreducible $R$-module and faithful since $N$ is a faithful $S$-module.

COROLlaRY 1.5. If $R$ is a prime von Neumann regular ring which -satisfies a generalized polynomial identity over its extended centroid $C$, then $R$ is primitive with a minimal right ideal $e R$ with $e^{2}=e$.

Proof. By Martindale [9], $S$ is primitive with minimal right ideal $e S$ where $e^{2}=e$. Therefore $R$ is primitive and $e S$ is a faithful irreducible $R$-module. Since $S$ is an essential extension of $R, 0 \neq e S \cap R \subseteq e S$ and so $e R=e S \cap R=e S$.

EXAMPLE 1.6. We give an example to show that the central closure of a prime von Neumann regular ring need not be regular. Let $F$ be a field of characteristic 2 and let $K=F\left(x^{2}\right), L=F(x)$. Then $L$ is an inseparable extension of $K$ and $[L: K]=2$. We embed $L$ in the $2 \times 2$ matrices over $K$. Let $R$ be the ring of $\boldsymbol{\aleph}_{0} \times \boldsymbol{\aleph}_{0}$ matrices in block diagonal form with $A_{2 n}$ the first block and $B$ repeated in the remainder of the blocks down the main diagonal where $A_{2 n}$ is a $2 n \times 2 n$ ( $n$ arbitrary) matrix with coefficients in $L$ and $B \in L \subseteq K_{2}$. Then $R$ is prime regular and the central closure of $R$ looks the same as $R$ except that it has elements of $L \otimes_{K} L$ repeated down the main diagonal. Consequently, $R C$ is not regular because it has the factor ring $L \otimes_{K} L$ which is not semisimple.

2. Locally Artin rings. From the study of group algebras of countable locally finite groups has come a class of rings which we shall call "locally Artinian" rings.

Definition. A ring $R$ is locally (left) Artinian if $R=\bigcup_{i=1}^{\infty} R_{i}$ where each $R_{i}$ is a left Artinian ring, $R_{1} \subseteq R_{2} \subseteq R_{3} \subseteq \cdots$, and the $R_{i}$ have a common unity element. 
ExAmples. (2.1) If $A$ is a left Artinian ring and $G$ is a countable locally finite group, then the group ring $A[G]$ is locally Artinian. See [8, p. 159].

(2.2) Any locally finite algebra over a field which has countable dimension, in particular, any countable dimensional algebraic algebra which satisfies a polynomial identity.

(2.3) Let $R$ be a left Artinian ring with unity and let $S$ be the ring of $\boldsymbol{\aleph}_{0} \times \boldsymbol{\aleph}_{0}$ row finite matrices with entries in $R$. If $T$ is the subring of $S$ which is generated by $R \cdot 1$ and all matrices with at most finitely many nonzero entries, then $T$ is locally Artinian.

A ring is said to be $\pi$-regular if for each $a$ in $R$, there exists an $x$ in $R$ and a positive integer $n$ such that $a^{n}=a^{n} x a^{n}$ [6]. That locally Artinian rings are $\pi$-regular follows from the fact that Artinian rings are $\pi$-regular. We include a proof for completeness.

\section{Lemma 2.1. If $R$ is locally Artinian, then $R$ is $\pi$-regular.}

Proof. If $a$ is in $R$, then $a$ is in $R_{i}$ for some $i$. Hence it will suffice to show that $R_{i}$ is $\pi$-regular. Since $R_{i}$ is left Artinian, it is semiprimary and thus both left and right perfect. By Bass [2] we have that $R_{i}$ satisfies the descending chain condition on both principal right ideals and principal left ideals. Therefore there exists a positive integer $n$ such that simultaneously $R_{i} a^{n}=R_{i} a^{2 n}$ and $a^{n} R_{i}=a^{2 n} R_{i}$, i.e., there exists an $x$ and $y$ in $R_{i}$ such that $a^{n}=x a^{2 n}$ and $a^{n}=a^{2 n} y$. In fine, $a^{2 n}=a^{2 n} y x a^{2 n}$ and $R_{i}$ is $\pi$-regular.

Remark. Let $R$ be a locally Artinian ring. Since $R$ is $\pi$-regular, it follows that $J(R)$ is nil and hence $J(R) \cap R_{i} \subseteq J\left(R_{i}\right)$. Then $J(R) \subseteq \bigcup_{i=1}^{\infty} J\left(R_{i}\right)$; however, they are not necessarily equal as can be seen by appropriately dissecting the $2 \times 2$ matrices over a field. In addition, $J(R)$ is locally nilpotent; however it need not be nilpotent as Example 2.8 shows.

THEOREM 2.2. Let $R$ be a semisimple locally Artinian ring. Then each nonzero right ideal of $R$ contains a nonzero idempotent and $R$ has a countable cofinal subset of ideals.

Proof. We have that each nonzero right ideal of $R$ contains a nonzero idempotent by Lemma 2.1. For each $i, R_{i}$ is semiprimary and hence $R_{i}$ has only finitely many nonzero idempotent ideals $\left\{I_{i j}: j=1,2, \cdots, k(i)\right\}$ by Alin and Armendariz [1] or Michler [10]. We claim that $\left\{R I_{i j} R\right.$ : $i=1,2,3, \cdots ; j=1,2, \cdots, k(i)\}$ is a cofinal subset of ideals of $R$. Let $J$ be a nonzero ideal of $R$. Then $J$ contains a nonzero idempotent. Hence there exists an $i$ such that $J \cap R_{i}$ contains a nonzero idempotent ideal of $R_{i}$. Let $B$ be a minimal nonnil ideal of $J \cap R_{i}$. Clearly $B^{2}$ is not nil and 
hence $B^{2}=B$ by the minimality of $B$. Therefore $B=I_{i j}$ for some $j$ and $R I_{i j} R \subseteq J$.

We have the following important corollary of Theorem 1.1.

Theorem 2.3. Let $R$ be a semisimple locally Artinian ring. Then $R$ is both left and right primitive if and only if $R$ is prime.

Proof. The result follows from Theorem 1.1 and Theorem 2.2.

Remark. The assumption that $R$ be semisimple is necessary as Examples 2.8 and 2.9 show. One immediate corollary is that locally Artinian rings enjoy the property of being left primitive if and only if they are right primitive.

The following corollaries of Theorem 2.3 relate it to the earlier work of Formanek and Snider [5] and Passman [11] on primitive group algebras. The first corollary extends Passman [11, Theorem 7].

Corollary 2.4. Let $A$ be a left Artinian ring and let $G$ be a countable locally finite group such that $A[G]$ is semisimple. Then $A[G]$ is both left and right primitive if and only if $A[G]$ is prime.

Proof. As was shown in Example 2.1, $A[G]$ is locally Artinian. Therefore the result follows immediately from Theorem 2.3.

Corollary 2.5 (Formanek-SNider). Let $G$ be a countable locally finite group and let $F$ be a field of characteristic zero, or of characteristic $p$ if $G$ has no elements of order $p$. Then $F[G]$ is primitive if and only if $F[G]$ is prime.

Proof. It follows from Maschke's theorem that $F[G]$ is semisimple. Therefore the result follows from Corollary 2.4.

Remark. In order to obtain this result Formanek and Snider [5] prove that prime "locally semisimple Artinian" rings, i.e., ones which are a union of an ascending sequence of semisimple Artinian rings are primitive. We note that "locally semisimple Artinian" rings are locally Artinian and semisimple but not conversely.

Lemma 2.6. Let $F$ be a field and let $G$ be a group. If $H$ is a subgroup of $G$ such that $F[H]$ is primitive and if each nonzero ideal of $F[G]$ has a nonzero contraction in $F[H]$, then $F[G]$ is primitive.

Proof. Since $F[H]$ is primitive, there exists a maximal right ideal $K$ of $F[H]$ which contains no nonzero ideals of $F[H]$. Now $K F[G]$ is a right ideal of $F[G]$ and it is proper since, if $\{e\} \cup\left\{x_{i}: i \in J\right\}$ is a complete set of right coset representatives of $H$ in $G$, then $K F[G]=K \oplus\left(\bigoplus_{i \in J} K x_{i}\right) \neq$ $F[G]$. Consequently, $K F[G]$ is contained in a maximal right ideal $M$ of 
$F[G]$. Moreover, if $M$ contains a nonzero ideal $I$ of $F[G]$, then $0 \neq I \cap$ $F[H] \subseteq M \cap F[H]=K$, contradiction. Therefore, $M$ contains no nonzero ideals of $F[G]$ and wherefore $F[G]$ is primitive.

THEOREM 2.7. Let $X$ be an infinite set and let $G$ be the group of all permutations of $X$ which move only finitely many elements of $X$. If $F$ is any field, then $F[G]$ is both left and right primitive.

Proof. Let $Y$ be a countably infinite subset of $X$ and let $H$ be the subgroup of $G$ consisting of all those permutations of $X$ which fix all the elements not in $Y$. Thus $H$ is a countable locally finite group. Moreover, $F[H]$ is prime by Connell $[3$, Theorem 8, p. 675] and $F[H]$ is semisimple by Formanek [4]. Therefore $F[H]$ is both left and right primitive by Corollary 2.4 .

The crux of the argument is to show that each nonzero ideal $J$ of $F[G]$ has a nonzero contraction in $F[H]$. Let $0 \neq a \in J$ and let $\left\{g_{i}: i=1,2, \cdots, k\right\}$ be the support of $a$. By looking at the cycle decomposition of the $g_{i}$, we see that there exists $g \in G$ with $g g_{i} g^{-1} \in H, i=1,2, \cdots, k$. Hence $0 \neq$ gag $^{-1} \in J \cap F[H]$. That $F[G]$ is both left and right primitive now follows from Lemma 2.6.

EXAMPLE 2.8. We give an example of a prime locally Artinian ring $R$ which is not semisimple. Let $F$ be a field and let $R$ be the subring of the ring of $\boldsymbol{\aleph}_{0} \times \boldsymbol{\aleph}_{0}$ row finite matrices over $F$ which have the following form: $r$ in $R$ if there exists an $n \times n$ upper triangular matrix $A$ in $F_{n}$ such that $r$ is in block diagonal form with $A$ repeated down the main diagonal and zeros elsewhere. First $R$ is locally Artinian, for let $R_{i}$ consist of all elements $r$ in $R$ for which there exists an $i \times i$ upper triangular matrix $A$ such that $r$ is in block diagonal form with $A$ repeated down the main diagonal. Then $R=\bigcup_{i=1}^{\infty} R_{i}$ and $R$ is not semisimple because $J(R)$ consists of all elements with zeros on the main diagonal. Finally we claim that $R$ is prime. Let $a$ and $b$ be nonzero elements of $R$. Then there exists $n \times n$ matrices $A$ and $B$ such that $a$ and $b$ are in block diagonal form with $A$ and $B$, respectively, repeated down the main diagonals. Since $F_{n}$ is prime, there exists $C$ in $F_{n}, C$ not necessarily upper triangular, with $A C B \neq 0$. Let $r$ in $R$ be the block diagonal matrix with $\left[\begin{array}{ll}0 & C \\ 0 & 0\end{array}\right]$ repeated down the main diagonal. Then $a r b$ is the nonzero block diagonal matrix with $\left[\begin{array}{ll}0 & A C B \\ 0 & \end{array}\right]$ repeated down the main diagonal. Consequently $R$ is prime.

EXAMPLE 2.9. For a group ring example of a prime locally Artinian ring which is not semisimple, let $F$ be a field of characteristic $p$ and let $G=\bigcup H_{i}$ where $H_{1}=Z_{p}$ and $H_{n+1}$ is the wreath product of $H_{n}$ by $Z_{p}$. It can be shown that $F[G]$ is a prime locally Artinian ring and $J(F[G])$ is the augmentation ideal since $G$ is a locally finite $p$-group. 
We wish to thank Edward Formanek for many valuable comments on the exposition, including Lemma 2.6 and Example 2.9.

\section{REFERENCES}

1. J. S. Alin and E. P. Armendariz, TTF-classes over perfect rings, J. Austral. Math. Soc. 11 (1970), 499-503. MR 43 \#258.

2. H. Bass, Finitistic dimension and a homological generalization of semiprimary rings, Trans. Amer. Math. Soc. 95 (1960), 466-488. MR 28 \#1212.

3. I. G. Connell, On the group ring, Canad. J. Math. 15 (1963), 650-685. MR 27 \#3666.

4. E. Formanek, A problem of Passman on semisimplicity, Bull. London Math. Soc. 4 (1972), 375-376.

5. E. Formanek and R. L. Snider, Primitive group rings, Proc. Amer. Math. Soc. 36 (1972), 357-360.

6. N. Jacobson, Structure of rings, 2nd ed., Amer. Math. Soc. Colloq. Publ., vol. 37, Amer. Math. Soc., Providence, R.I., 1964. MR 36 \#5158.

7. I. Kaplansky, Algebraic and analytic aspects of operator algebras, Amer. Math. Soc., Providence, R.I., 1970.

8. J. Lambek, Lectures on rings and modules, Blaisdell, Waltham, Mass., 1966. MR 34 \#5857.

9. W. S. Martindale III, Prime rings satisfying a generalized polynomial identity, J. Algebra 12 (1969), 576-584. MR 39 \#257.

10. G. Michler, Idempotent ideals in perfect rings, Canad. J. Math. 21 (1969), 301-309. MR 39 \#245.

11. D. S. Passman, Primitive group rings, Pacific J. Math. 47 (1973), 499-506.

Department of Mathematics, University of Texas, Austin, Texas 78712

Department of Mathematics, Northwestern University, Evanston, Illinois 60201 